PROCEEDINGS OF THE

AMERICAN MATHEMATICAL SOCIETY

Volume 86, Number 4, December 1982

\title{
UNSTABLE WEAK ATTRACTORS
}

\author{
RONALD A. KNIGHT
}

\begin{abstract}
Our objective in this paper is to continue the process of classification and characterization of weak attractors initiated by the author in an earlier paper. In particular, we obtain additional characterizations of those weak attractors which are saddle sets and bilateral weak attractors.
\end{abstract}

1. Introduction. Considerable effort has been directed toward the characterization of a flow near an arbitrary set. Compact minimal sets, compact invariant sets, and closed positively invariant sets with compact boundaries on locally compact Hausdorff phase spaces have received most of the attention. Even for those sets a complete categorization of all possible types of flows is an enormous task which may never be completed. This paper will focus primarily on characterizations of unstable flows near closed weak attractors with compact boundaries on locally compact Hausdorff phase spaces. In [4] the author characterized such stable, saddle, and bilateral weak attractors. We shall obtain additional characterizations of saddle weak attractors and bilateral weak attractors.

A given dynamical system or continuous flow $(X, \pi)$ on a Hausdorff phase space $X$ will be assumed throughout this paper. The symbols $C, K$ and $L$ denote, respectively, the trajectory, orbit closure and limit set relations. The unilateral versions of these relations carry the appropriate superscript + or - . A point $x$ of $X$ is positively (weakly) attracted to a set $M \subset X$ if the net $(x t), t>0$, is ultimately (frequently) contained in each neighborhood of $M$. The region of positive (weak) attraction for a set $M \subset X$ is denoted by $A^{+}(M)\left(A_{w}^{+}(M)\right)$. We say that a set $M \subset X$ is a positive (weak) attractor whenever $A^{+}(M)\left(A_{w}^{+}(M)\right)$ is a neighborhood of $M$. Frequently, the adjective "positive" is omitted. The following subclasses of attractors were identified in [4]. A (weak) attractor $M \subset X$ is called recursive provided the net $(x t), t>0$, is ultimately (frequently) contained in $M$ for each point $x$ of $A^{+}(M)\left(A_{w}^{+}(M)\right)$. A set $M$ is said to strongly attract a point $x$ of $X$ if for each neighborhood $U$ of $M$ there exists a neighborhood $V$ of $x$ such that the net $(V t)$, $t>0$, is ultimately contained in $U$. The region of strong attraction is denoted by $A_{s}^{+}(M)$. The negative versions of each of the attraction notions above are defined and denoted in the obvious manner. Finally, a set $M \subset X$ is called a saddle set if and only if there exists a neighborhood $U$ of $M$ such that every neighborhood $V$ of $M$ contains at least one point $x$ with $C^{+}(x) \not \subset U$ and $C^{-}(x) \not \subset U$.

We denote the closure, interior, boundary, and complement of a set $M \subset X$ by $\bar{M}, M^{0}, \partial M$ and $X \backslash M$, respectively. The set of nonnegative (nonpositive) real numbers is denoted by $R^{+}\left(R^{-}\right)$.

Received by the editors January 11, 1982.

1980 Mathematics Subject Classification. Primary 34C35; Secondary 54H20.

Key words and phrases. Attractor, dynamical system, flow, recursive attractor, recursive weak attractor, saddle set, stable, strong attraction, weak attractor.

(c) 1982 American Mathematical Society 0002-9939/82/0000-0290/\$02.25 
Each of the basic properties of dynamical system theory used in this paper are presented in detail in references [1, 2 and 3].

2. Unstable weak attractors. The major characterizations related to this paper obtained in reference [4] by the author are stated in the following theorem:

THEOREM. Let $M$ be a closed weak attractor with compact boundary and let $X$ be locally compact. Then

(a) $M$ is stable if and only if it is positively invariant and satisfies Zubov's condition, i.e. $L^{-}(X \backslash M) \cap M=\varnothing$;

(b) $M$ is a saddle set if and only if $A_{w}^{+}(M) \backslash M \neq A_{s}^{+}(M) \backslash M$ and

(c) $M$ is a bilateral weak attractor if and only if $A_{w}^{+}(M) \backslash M=A_{w}^{-}(M) \backslash M$.

COROLLARY. Let $M$ be a closed weak attractor with compact boundary and let $X$ be locally compact. Then, exactly one of the following holds:

(a) $M$ is stable.

(b) $M$ is a saddle set.

(c) $M$ is a nonsaddle set and some point of $M$ is not strongly attracted to $M$.

Our objective in each of the following results is to examine more closely saddle and bilateral weak attractors. The first theorem is a very straightforward and simple characterization of these classes of sets where $M$ and $X$ are arbitrary. Most often the sets considered are compact but following Theorem 1 and its corollary we give examples showing that the characterization of part (a) cannot be strengthened by omitting either condition (i) or (ii) even when both the set and the phase space are compact. Moreover, the same examples also illustrate the sharpness of the characterization of part (b) in this case.

For a point $x$ in $M$ the number of times $C(x)$ leaves $M$ is the cardinality of the class of components of $\{t: x t \in C(x) \backslash M\}$.

THEOREM 1. A weak attractor $M$ is a

(a) saddle set if and only if either

(i) some trajectory leaves $M$ at least twice or else

(ii) each trajectory leaves $M$ at most once and there is a neighborhood $U$ of $M$ such that each neighborhood of $M$ intersects some trajectory leaving $U$ at least twice,

(b) bilateral weak attractor if and only if for each neighborhood $U$ of $M$ and each point $x$ in $A_{w}^{+}(M) \backslash M$ the negative semitrajectory through $x$ intersects $U$.

PROOF. Since statement (b) is an obvious result of the definitions of positive and negative weak attraction, we shall consider statement (a). Let $M$ be a saddle set such that condition (i) does not hold. Then, trivially condition (ii) holds because the existence of a neighborhood $U$ like the one described in (ii) is always guaranteed by the definition of a saddle set. Conversely, if condition (ii) holds, then $M$ is necessarily a saddle set. Moreover, if condition (i) holds, then there is a point $x$ in $M$ such that $x t_{0}$ and $x t_{1}$ are in $X \backslash M$ for some $t_{0}<0$ and some $t_{1}>0$. Letting $U=$ $X \backslash\left\{x t_{0}, x t_{1}\right\}$ we have $C^{-}(x) \not \subset U, C^{+}(x) \not \subset U$, and $x \in V$ for each neighborhood $V$ of $M$. Hence, $M$ is a saddle set and the proof is complete.

Each point of the region of weak attraction for a set $M$ has its positive semitrajectory entering $M$ provided $M$ is a recursive weak attractor. Thus, statement (b) can be revised as follows. We say $C^{+}(x)\left(C^{-}(x)\right)$ is frequently in $M$ if $\left(x t_{i}\right)$ is in $M$ for some net $t_{i} \rightarrow+\infty\left(t_{i} \rightarrow-\infty\right)$. 
COROLlARY 1.1. Let $M$ be a recursive weak attractor. Then, $M$ is a bilateral weak attractor if and only if each negative semitrajectory leaving $M$ returns to each neighborhood of $M$ frequently often in the negative direction.

COROLLARY 1.2. Every recursive weak attractor which is iot a recursive attractor is a saddle set.

Examples of saddle sets satisfying the criteria of part (a) conditions (i) and (ii) are easily constructed. Even if the set is compact (and a recursive weak attractor) and the phase space is compact, both conditions can be realized as the following examples illustrate.

EXAMPLES. (1) Let the phase space consist of exactly one periodic noncritical orbit. Any singleton set is a bilateral recursive weak attractor satisfying part (a) condition (i) of Theorem 1.

(2) Consider the planar flow defined by $\dot{r}=r(1-r), \dot{\theta}=\sin ^{2} \theta / 2$ of Example 1.7.5 on p. 59 of [3]. Let $(X, \pi)$ be the extended flow induced on the one point compactification of the phase plane. Then, the compact half-disc $M=\{(r, \theta)$ : $2 \cos \theta \leq r \leq 0, \pi / 2 \leq \theta \leq \pi\}$ is a saddle recursive attractor such that no trajectory leaves $M$ more than once and condition (ii) holds. Also, $M \cup\{\infty\}$ is a compact saddle bilateral recursive attractor.

Some recursive weak attractors never satisfy part (a) condition (ii) of Theorem 1. The succeeding theorem addresses such a class of weak attractors.

THEOREM 2. Let $M$ be a closed recursive weak attractor on a locally compact phase space $X$ and let the boundary of $M$ contain no semitrajectory. Then, $M$ is a saddle set if and only if some trajectory leaves $M$ at least twice.

PROOF. The statement will follow from Theorem 1 if we show that condition (ii) cannot hold when $M$ is a saddle set. Suppose that $M$ is a saddle set for which each trajectory leaves $M$ at most once. Choose the neighborhood $U$ of $M$ contained in $A_{w}^{+}(M)$ so that $U \backslash M^{0}$ is compact. There is a net $\left(x_{i}\right)$ in $U$ converging to a point $x$ in $\partial M$ such that $C^{+}\left(x_{i}\right) \cap \partial U \neq \varnothing$ and $C^{-}\left(x_{i}\right) \cap \partial U \neq \varnothing$ for each $i$. Define $T_{y}=$ $\inf \left\{t \in R^{+}: y t \in M^{0}\right\}$ for each point $y$ in $A_{w}^{+}(M)$. For each point $y$ in $A_{w}^{+}(M)$ we have $C^{+}(y) \cap M^{0} \neq \varnothing$ because $C(y) \backslash M$ is connected, $\partial M$ contains no semitrajectory, and no periodic orbit can be in $A_{w}^{+}(M) \backslash M^{0}$. Define $T=\sup \left\{T_{y}: y \in U\right\}$ and we shall show that $T<+\infty$. For every point $y$ in $U$ there exists an open neighborhood $V_{y}$ of $y$ such that $V_{y} t \subset M^{0}$ for some $t$ between $T_{y}$ and $T_{y}+1$ by the continuity of $\pi$. Note that for any point $y$ of $U, T_{p} \leq T_{y}+1$ for every point $p$ in $V_{y}$. The set $\left\{V_{y}: y \in U \backslash M^{0}\right\}$ is an open cover of the compact set $U \backslash M^{0}$, and hence, contains a finite subcover $\left\{V_{y_{1}}, \ldots, V_{y_{n}}\right\}$ of $U \backslash M^{0}$. Therefore, $T \leq \max \left\{T_{y_{i}}+1: 1 \leq i \leq n\right\}<$ $+\infty$. Now, if $C^{+}\left(x_{i} T\right) \cap \partial U \neq \varnothing$ for some $i$, then $C^{+}\left(x_{i}\right)$ contains a point of $M$ and $C\left(x_{i}\right)$ leaves $M$ in both directions which is impossible. Thus, there is a net $\left(t_{i}\right)$ in $R^{+}$such that $x_{i} t_{i}$ is in $\partial U$ and $t_{i} \leq T$. Some subnet $\left(t_{n_{i}}\right)$ of $\left(t_{i}\right)$ converges to a point $\tau \leq T$, and hence, $x_{n_{i}} t_{n_{i}} \rightarrow x \tau$ in $\partial U$. The semitrajectory $C^{+}(x)$ intersects $\partial U$. Moreover, the dual argument implies that $C^{-}(x)$ also intersects $\partial U$ which is absurd. Consequently, some trajectory leaves $M$ at least twice. The proof is complete.

COROLLARY 2.1. Let $M$ be a closed recursive weak attractor with compact boundary containing no semitrajectory on a locally compact phase space $X$. Exactly one of the following holds: 
(a) $M$ is positively invariant ( $M$ is stable).

(b) Some trajectory leaves $M$ more than once ( $M$ is a saddle set).

(c) No trajectory leaves $M$ more than once and some point outside of $M$ is bilaterally weakly attracted to $M(M$ is an unstable nonsaddle set).

The following theorem characterizes closed bilateral weak attractors with compact boundaries.

THEOREM 3. Let $M$ be a closed weak attractor with compact boundary on a locally compact phase space $X$. Then, $M$ is a bilateral weak attractor if and only if for each neighborhood $V$ of $M$ contained in $A_{w}^{+}(M)$ there is a $T_{V}>0$ such that $A_{w}^{+}(M)=V\left[0, T_{V}\right]$.

Proof. Let $M$ be a bilateral weak attractor and select $V$ as indicated in the hypothesis. Let $U$ be a neighborhood of $M$ contained in $V$ such that $U \backslash M^{0}$ is compact. Just as in the proof of Theorem 2 we can find a $\tau>0$ such that $\tau=$ $\sup \left\{T_{y}: y \in U\right\}<+\infty$ where $T_{y}=\inf \left\{t \in R^{+}: y t \in U^{0}\right\}$. For any point $x$ in $A_{w}^{+}(M) \backslash U$ let $\sigma=\sup \left\{t \in R^{-}: x t \in U\right\}$. Then, $x \sigma \in \partial U$ and $x(\sigma, 0] \cap U=\varnothing$. Now, $x \in x \sigma[0, \tau] \subset U[0, \tau]$, and therefore, $A_{w}^{+}(M)=U[0, \tau]$. By letting $T_{V}=\tau$ we have the desired equation. Conversely, if $M$ is a unilateral weak attractor, then for some neighborhood $V$ of $M$ and some point $x$ in $V, C^{-}(x) \backslash V$ contains $C^{-}(x t)$ for some $t<0$. Thus, $x\left(t-T_{V}\right)\left[0, T_{V}\right]=x t\left[-T_{V}, 0\right] \subset C^{-}(x t)$ so that $x\left(t-T_{V}\right) \notin V\left[0, T_{V}\right]=$ $A_{w}^{+}(M)$. Since this is impossible, $M$ must be a bilateral weak attractor. The proof is complete.

Note that the neighborhood $V$ of Theorem 3 is a recursive weak attractor. Whenever $M$ is a recursive weak attractor and each trajectory attracted to $M$ intersects the interior of $M$, then Theorem 3 can be strengthened as follows.

THEOREM 4. Let $M$ be a closed recursive weak attractor with compact boundary on a locally compact phase space $X$ such that $A_{w}^{+}(M)=A_{w}^{+}\left(M^{0}\right)$. Then, $M$ is a bilateral weak attractor if and only if $A_{w}^{+}(M)=M[0, T]$ for some $T>0$.

PrOOF. First, we assume that $M$ is a bilateral weak attractor. By appropriately modifying the proof of Theorem 3 the condition $A_{w}^{+}(M)=M[0, T]$ for some $T>$ 0 is obtained. We need only define $T_{y}$ by $\inf \left\{t \in R^{+}: y t \in M^{0}\right\}$ instead of $\inf \left\{t \in R^{+}: y t \in U^{0}\right\}$ and consider again $\tau=\sup \left\{T_{y}: y \in U\right\}$. For any point $y$ in $A_{w}^{-}(M)=A_{w}^{+}(M)$ there is a point $z$ in $L^{-}(y) \cap M$. Since $z$ belongs to $A_{w}^{+}(M)$, we have $\varnothing \neq C^{+}(z) \cap M^{0} \subset L^{-}(y) \cap M^{0}$, and hence, $C^{-}(y) \cap M^{0} \neq \varnothing$. Consequently, $A_{w}^{-}(M)=A_{w}^{-}\left(M^{0}\right)$ and there is a dual $\tau^{\prime}>-\infty$ to the number $\tau$ defined above. Then, for the point $x$ selected in $A_{w}^{+}(M) \backslash U$ and for $\sigma$ as defined in the proof of Theorem 3 we have $\varnothing \neq x \sigma[0, \tau] \cap M=x[\sigma, \tau+\sigma] \cap M \subset x[0, \tau] \cap M$. Similarly, $\varnothing \neq x\left[\tau^{\prime}, 0\right] \cap M$. Choosing $T=\tau-\tau^{\prime}$ we obtain $A_{w}^{+}(M)=M[0, T]$. The converse follows in the same manner as in Theorem 3 completing the proof.

The requirement $A_{w}^{+}(M)=A_{w}^{+}\left(M^{0}\right)$ cannot be omitted from Theorem 4. To see this consider the example by Sullivan in [5] of a compact 5-manifold such that every orbit is periodic but the fundamental periods are unbounded. One can use this example to construct a closed recursive bilateral weak attractor $M$ with compact boundary such that $A_{w}^{+}(M) \neq A_{w}^{+}\left(M^{0}\right)$ and $A_{w}^{+}(M) \neq M[0, T]$ for each $T>0$. We need only construct $M$ so that there are periodic orbits with unbounded fundamental periods such that each is contained in $X \backslash M^{0}$ and each intersects 
$\partial M$ as well as $X \backslash \bar{M}$. Since Sullivan's example requires a lengthy discussion, the description of the example we assert the existence of would take an excessive amount of space, and hence, it is left to the interested reader to explicitly construct.

\section{REFERENCES}

1. N. Bhatia and O. Hajek, Theory of dynamical systems. Parts I and II, Tech. Notes BN-599 and BN-606, Univ. of Maryland, 1969.

2. __ Local semi-dynamical systems, Lecture Notes in Math., vol. 90, Springer-Verlag, Berlin and New York, 1969.

3. N. Bhatia and G. Szego, Stability theory of dynamical systems, Springer-Verlag, Berlin and New York, 1970.

4. R. Knight, Zubov's condition revisited, Proc. Edinburgh Math. Soc. (to appear).

5. D. Sullivan, A counterexample to the periodic orbit conjecture, Inst. Hautes Études Sci. Publ. Math. 46 (1976), 5-14.

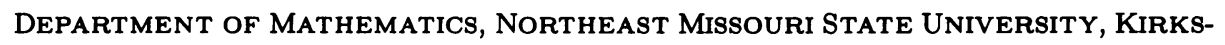
VILLE, MISSOURI 63501 